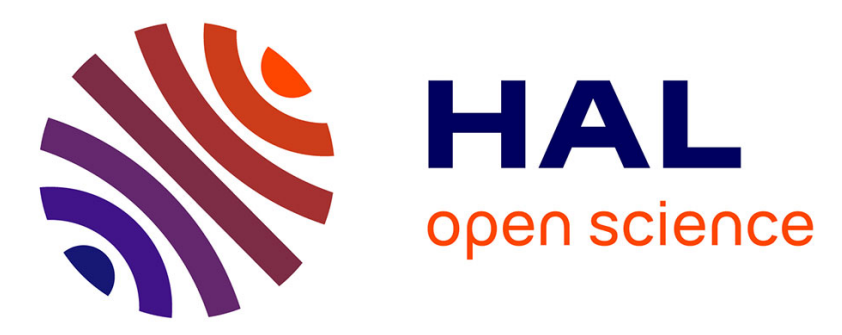

\title{
Superconducting Scanning Tunneling Microscope Tip to Reveal Sub-millielectronvolt Magnetic Energy Variations on Surfaces
}

\author{
Cristina Mier, Benjamin Verlhac, Léo Garnier, Roberto Robles, Laurent \\ Limot, Nicolás Lorente, Deung-Jang Choi
}

\section{To cite this version:}

Cristina Mier, Benjamin Verlhac, Léo Garnier, Roberto Robles, Laurent Limot, et al.. Superconducting Scanning Tunneling Microscope Tip to Reveal Sub-millielectronvolt Magnetic Energy Variations on Surfaces. Journal of Physical Chemistry Letters, 2021, 12 (11), pp.2983-2989. 10.1021/acs.jpclett.1c00328 . hal-03375139

\author{
HAL Id: hal-03375139 \\ https://hal.science/hal-03375139
}

Submitted on 12 Oct 2021

HAL is a multi-disciplinary open access archive for the deposit and dissemination of scientific research documents, whether they are published or not. The documents may come from teaching and research institutions in France or abroad, or from public or private research centers.
L'archive ouverte pluridisciplinaire HAL, est destinée au dépôt et à la diffusion de documents scientifiques de niveau recherche, publiés ou non, émanant des établissements d'enseignement et de recherche français ou étrangers, des laboratoires publics ou privés. 


\title{
A superconducting STM tip to reveal sub-meV magnetic energy variations on surfaces
}

\author{
Cristina Mier, ${ }^{\dagger}$ Benjamin Verlhac, ${ }^{\ddagger}$ Léo Garnier, ${ }^{\ddagger}$ Roberto Robles, ${ }^{\dagger}$ Laurent \\ Limot, ${ }^{\ddagger}$ Nicolás Lorente, ${ }^{\dagger,}$ and Deung-Jang Choi ${ }^{*, \dagger, \uparrow, \S}$ \\ $\dagger$ †entro de Física de Materiales CFM/MPC (CSIC-UPV/EHU), 20018 Donostia-San \\ Sebastián, Spain \\ $\ddagger$ Université de Strasbourg CNRS, IPCMS, UMR 7504, F-67000 Strasbourg, France \\ IDonostia International Physics Center (DIPC), 20018 Donostia-San Sebastián, Spain \\ §Ikerbasque, Basque Foundation for Science, 48013 Bilbao, Spain \\ E-mail: deungjang.choi@ehu.eus
}

February 22, 2021

\begin{abstract}
Combining the complex ordering ability of molecules with their local magnetic properties is a little-explored technique to tailor spin structures on surfaces. However, revealing the molecular geometry can be demanding. Nickelocene $(\mathrm{Nc})$ molecules present a large spin-flip excitation leading to clear changes of conductance at the excitationthreshold bias. Using a superconducting tip, we have the energy resolution to detect small shifts of the Nc spin-flip excitation thresholds, permitting us to reveal the different individual environment of Nc molecules in an ordered layer. This knowledge allows us to reveal the adsorption configuration of a complex molecular structure formed by Nc molecules in different orientations and positions. As a consequence, we infer that Nc layers present a strong non-collinear magnetic moment arrangement.
\end{abstract}


Revealing the intricate relation between magnetism and chemistry is a difficult problem that needs special probes sensitive to both magnetic and chemical interactions. This is of particular interest given the developing importance of two-dimensional arrays of magnetic moments. ${ }^{1}$ These arrays have a strong impact on the fields of magnetic domains, non-collinear magnetism and special spin structures such as skyrmions that are topologically protected. ${ }^{2,3}$ Most of these systems include magnetic interactions like exchange, Dzyaloshinky-Moriya and Ruderman-Kittel-Kasuya-Yoshida to create magnetic patterns of increasing sophistication. ${ }^{3-6}$ However, using molecular chemistry to pattern concrete magnetic arrangements is a different strategy. ${ }^{7,8}$ Molecules have ligand fields and local spin-orbit interactions that lead to sizeable magnetic anisotropy energies (MAE). ${ }^{9}$ This anisotropy implies preferential magnetic-moment directions and the appearance of molecular magnets. ${ }^{10}$ In the case of single molecules, the spin levels are discrete and properly, there is no energy barrier to turn the spin because the magnetic moment takes on discrete values, but rather excitation energies due to the energy difference between the spin states. Introducing then a hierarchical molecular structure via weak intermolecular interactions would add control and lead to rich patterns of magnetic arrays. ${ }^{11,12}$ Weak interactions can range from hydrogen and halogen bonds to weak electrostatic interactions. Among these, van der Waals (vdW) forces are particularly important in molecule-molecule and molecule-substrate interactions. On a free-electron-like surfaces, vdW interactions can shape the actual geometry of the potential energy surface describing surface chemistry. ${ }^{13} \mathrm{On} \mathrm{Pb}(111)$, vdW interactions are the main contribution to molecular adsorption. ${ }^{14-17}$ Complex molecular-magnet patterns on $\mathrm{Pb}$ can be expected.

Scanning probes have proved to be particularly powerful in monitoring and characterizing the on-surface synthesis of molecular structures, ${ }^{18-20}$ in particular when inelastic electron tunneling spectroscopy (IETS) is combined with the scanning tunneling microscope (STM). ${ }^{21-23}$ The excitation of a molecule by tunneling electrons leads to measurable changes in conductance that reveal the chemical identity of the molecular target. ${ }^{24,25}$ IETS thus becomes an important tool to determine the adsorption geometry of molecules thanks to the 
change in molecular-substrate interactions affecting the vibrational spectra. ${ }^{26,27}$ Magnetic IETS reveals a smaller-energy range of interactions ${ }^{28-30}$ and results in excitations that are usually lower and well distinct from vibrational ones. Its excitation efficiency is however one order of magnitude bigger, and is then interesting to study the variation of magnetic IETS with molecular interactions. ${ }^{31,32}$

Here, we use the enhanced energy resolution of a superconducting STM tip ${ }^{33-36}$ to reveal the small magnetic changes on a molecular-magnet layer. We focus our attention onto a prototypical molecular magnet, nickelocene $(\mathrm{Nc})$, and on the vdW-governed arrays it forms on $\mathrm{Pb}$ (111). The inset of Fig. 1 (a) shows that Nc is composed by two cyclopentadienyl rings $\left(\mathrm{C}_{5} \mathrm{H}_{5}\right)$ and a $\mathrm{Ni}$ atom in between. It is a $S=1$ spin with hard-axis longitudinal magnetic anisotropy energy (MAE) perpendicular to the cyclopentadienyl rings that ranges from $D=3.4$ to $4.0 \mathrm{meV}$ depending on the substrate. ${ }^{32,37-39}$ In order to reveal the connection between chemical and magnetic interactions, we first adsorb a molecule on the tip and study the changes in MAE through the variations of magnetic IETS as a function of the interaction between molecule and the $\mathrm{Pb}$ (111) surface. This allows us to characterize each molecule of a Nc layer when we study their MAE changes as a function of their position in the molecular layer.

Nickelocene presents very large changes in conductance associated to the inelastic $S_{z}=0$ to $S_{z}= \pm 1$ transition induced by tunneling electrons (inset Fig.2 (e)). ${ }^{37}$ Studies on copper surfaces $^{37,38}$ show that the changes in conductance can be as large as $900 \%$ becoming one of the strongest IETS signals to date. In order to detect thresholds shifts in the $\mu \mathrm{eV}$-range, we take advantage of the quasiparticle (QP) peaks present in the density of states (DOS) of the superconducting tip, that render small shifts easy to detect (see below).

Figure 1 (a) shows a constant current STM image of the Pb (111) surface taken with Nc at the tip apex of the superconducting tip. The molecular tip gives an enhanced spatial resolution ${ }^{33-36}$ that permits us to discriminate among top (black dot), hollow (red and green dots) and bridge (orange dot) sites. To further distinguish HCP from FCC hollow sites, we 

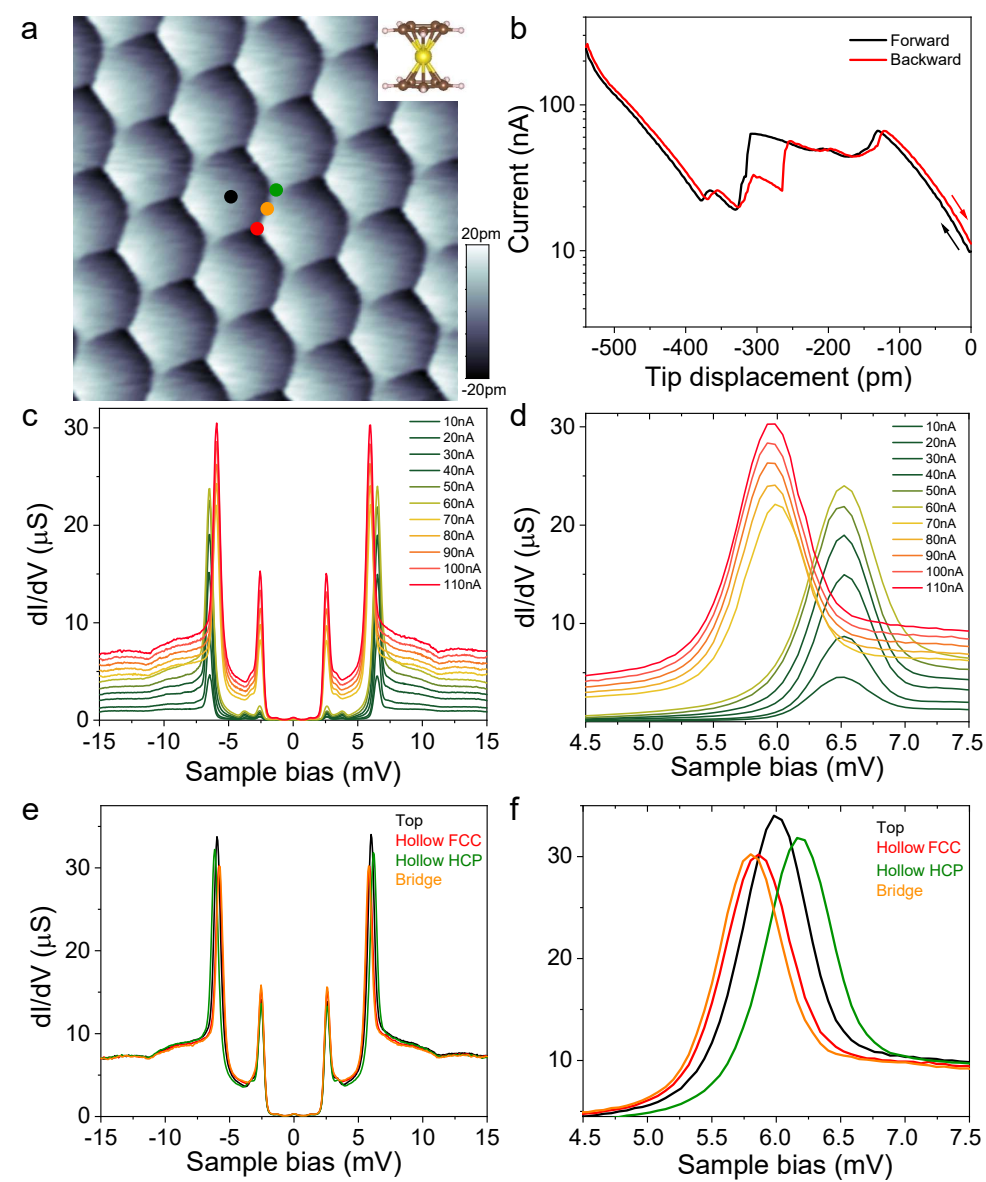

Figure 1: Molecular tip sensing surface interactions. (a) Atomically resolved constant current image marking the four lateral sites where the molecular tip will probe the surface $(-15$ $\mathrm{mV}, 70 \mathrm{pA}, 1.5 \times 1.5 \mathrm{~nm}^{2}$, the gray scale goes from -20 to $20 \mathrm{pm}$ depicting the height corrugation). Inset: the Nc molecule consists of a Ni atom (yellow) capped by $\mathrm{C}$ (brown) pentagons (or cyclopentadienyls) with H-saturated bonds (white), the gas-phase distance between cyclopentadienyls is $3.6 \AA$. (b) Current versus tip displacement for a fixed sample bias of $15 \mathrm{mV}$, where the tip-displacement origin is taken at $10 \mathrm{nA}$. The black line shows the evolution of the current when reducing the surface-molecule distance. The red line corresponds to the inverse path. (c) The $d I / d V$ spectra between -15 and $15 \mathrm{mV}$ measured on top of a $\mathrm{Pb}$ atom showing a clear shift of the inelastic peak when the molecule is close enough to the surface. (d) Same as (c) but reducing the plotted bias range to reveal the sharp shift at about $70 \mathrm{nA}$. Beyond this transition, the peak positions evolve very slowly with increasing currents but they depend on the tip's locations with respect to surface site. (e) At fixed tip-displacement of current $100 \mathrm{nA}$ and sample bias $15 \mathrm{mV}$, the $d I / d V$ spectra were measured at different adsorption sites; ontop a $\mathrm{Pb}$ surface atom (black), on the HCP hollow site (red), on the bridge site (orange) and on the FCC hollow site (green) showing shifts from IETS peak at $6.50 \mathrm{meV}$ in the tunneling regime (d). (f) same as (e) but reducing the plotted bias range to reveal the IETS peak shifts at different adsorption sites. 

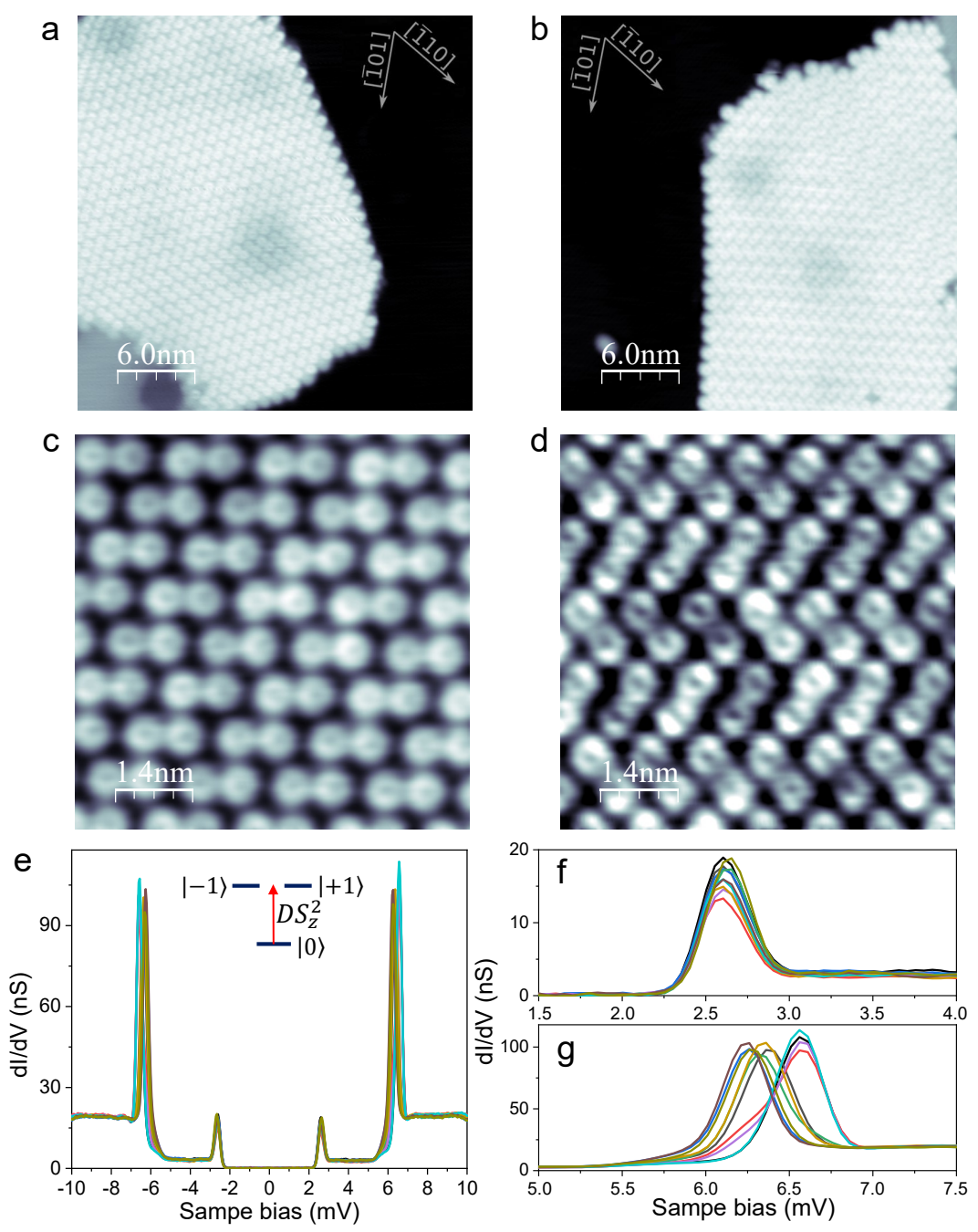

Figure 2: Molecular islands on $\mathrm{Pb}$ (111) studied with a superconducting tip. (a) Constantcurrent image of an island of $\mathrm{Nc}$ on $\mathrm{Pb}(111)$ where the $\mathrm{Nc}$ dimers are aligned $(-60 \mathrm{mV}$, $\left.20 \mathrm{pA}, 30 \times 30 \mathrm{~nm}^{2}\right)$. (b) Constant-current image of Nc dimers arranged in zig-zag $(-80$ $\mathrm{mV}, 40 \mathrm{pA}, 30 \times 30 \mathrm{~nm}^{2}$ ). (c) The paired arrangement of $\mathrm{Nc}$ dimers is evidenced at shorter tip-surface distances $\left(-20 \mathrm{mV}, 20 \mathrm{pA}, 7.0 \times 7.0 \mathrm{~nm}^{2}\right)$. (d) The zig-zag arrangement is clearly seen at shorter tip-surface distances $\left(-20 \mathrm{mV}, 20 \mathrm{pA}, 7.0 \times 7.0 \mathrm{~nm}^{2}\right)$. (e) Tunnel $d I / d V$ spectra obtained with a superconducting tip over several molecules at different locations. (f) The quasiparticle (QP) peak consistently appears at $2.61 \pm 0.02 \mathrm{mV}$, but $(\mathrm{g})$ the inelastic peak depends on the measured molecules (the uncertainty has been determined from the variance of the measurements over 45 molecules). 
observe a larger contribution to the current on the HCP site due to the atom situated below it. ${ }^{40}$ This is a usual constant-current map, where the apparent three-dimensional plot is due to the difference between HCP and FCC sites.

The evolution of the tunneling current $(I)$ with tip-substrate distance $(z)$ yields interesting information on the tip-substrate interactions. As the molecule approaches the surface, Fig. 1 (b), the current exponentially increases with distance. At a current about $70 \mathrm{nA}$ at $15 \mathrm{mV}$, or $214 k \Omega$ of junction resistance, the interaction with the surface is strong enough to create instabilities in the molecular junction, stopping the exponential increase of the current. Approaching and retracting the tip leads to a hysteretic behavior implying molecular rearrangements as the interactions change. The small lateral offset is caused by a delay in data acquisition. The $I(z)$ curve is highly reproducible for each set of measurements and we infer that the molecular tip is changing between precise local minima with $z$.

Measurements are carried out at a base temperature of $2.5 \mathrm{~K}$. The STM tip is coated by $\mathrm{Pb}$ giving a superconducting gap slightly smaller than the bulk $\mathrm{Pb}$ gap of $\Delta=1.35 \mathrm{meV},{ }^{41}$ due to finite-size and temperature effects. ${ }^{42}$ The surface superconducting gap appears at $\Delta_{\text {tip }}+\Delta_{\text {substrate }} \approx 2 \Delta$ in the $d I / d V$ spectra, due to the convolution with the tip electronic structure. ${ }^{33-36}$ This is confirmed by the two QP peaks appearing at $\pm 2.55 \pm 0.02 \mathrm{mV}$ in Fig. 1 (c). The bias values of the QP peaks do not depend on the tip's vertical and lateral distance to the surface. However, when $\mathrm{Nc}$ is present on the superconducting tip, an additional structure is present at about $6.0 \mathrm{mV}$ which depends on the tip-surface distance, Fig. 1 (c)(d), and lateral location, Fig. 1 (e)-(f). These peaks, Fig. 1 (f) show the IETS enhancement across the molecular excitation threshold. The excitation bias is shifted by $2 \Delta$ yielding and excitation energy of $D=6.5-2.55 \pm 0.2=3.95 \pm 0.2 \mathrm{meV}$, due to the presence of the tip and substrate superconducting gaps. ${ }^{43}$

Recording the differential conductance at different molecule-surface distances gives a jump in the bias of the IETS peaks at about $70 \mathrm{nA}$ (junction resistance $214 k \Omega$ ). Figure 1 (c) shows that the IETS peak are constant except for a fast transition about $70 \mathrm{nA}$, with 
only two values for the IETS peak position as revealed when zooming in the figure, Fig. 1 (d). From Fig. 1 (b) we see that there are two limiting molecular conformations at $70 \mathrm{nA}$. Arguably, the molecule is changing while the tip is approaching from -120 pm to $-400 \mathrm{pm}$, but after this evolution the reproducibility of the $I(z)$ curves and of the conductance indicates that the molecule has two distinct conformations, one in the approaching sequence until -120 pm and another one passed $-400 \mathrm{pm}$. This explains why two values for the IETS peaks are found as the molecule approaches or retracts from the surface. The local properties of the molecule are different in these two conformations leading to different MAE. ${ }^{37}$

The molecule-substrate interactions become larger as the tip approaches the surface. Yet, only a small variation of $0.03 \mathrm{meV}$ in the excitation threshold is observed as the current increases from $70 \mathrm{nA}$ to $110 \mathrm{nA}$, Fig. 1 (d). This is very different compared to copper surfaces, ${ }^{38,44}$ where changes in the molecule-substrate interaction reduce the molecular spin. In the present case, the molecule-substrate interaction is visibly not big enough to drive the change of the molecular spin. The lateral dependence shows a stronger evolution. At 100 nA, Fig. 1 (e), the molecule ontop a Pb surface atom (black) presents a shift of $0.52 \mathrm{meV}$, on the FCC hollow site (red) the shift is $0.65 \mathrm{meV}$, on the bridge site (orange) the shift is $0.70 \mathrm{meV}$ and on the HCP hollow site (green) the shift is $0.34 \mathrm{meV}$ with respect to the 6.50 meV IETS peak for currents below $70 \mathrm{nA}$ in Fig. 1 (d).

These data show very interesting features. Overall there is an average shift of $\sim 0.5$ meV due to the new conformation of the molecule on the tip as revealed by the jump at 70 nA. Additionally, the local interaction with the substrate induces a small shift ranging from 0.3 to $0.7 \mathrm{meV}$, revealing the lateral position of the molecule with respect to the surface. Due to the small coordination of the molecule with the tip's atoms, we expect that once the molecule is stable, the tip-molecule interactions slightly change. $\mathrm{Nc}$ is fairly rigid in regards to compression, showing a very small shift as the molecule is compressed, except when a major molecular change takes place. This amounts to the first shift. Laterally, our calculations of projected density of states on molecular orbitals, ${ }^{45}$ show that there are small 
variations of charge transfer due to the change of molecular location, leading to the changes with surface site.
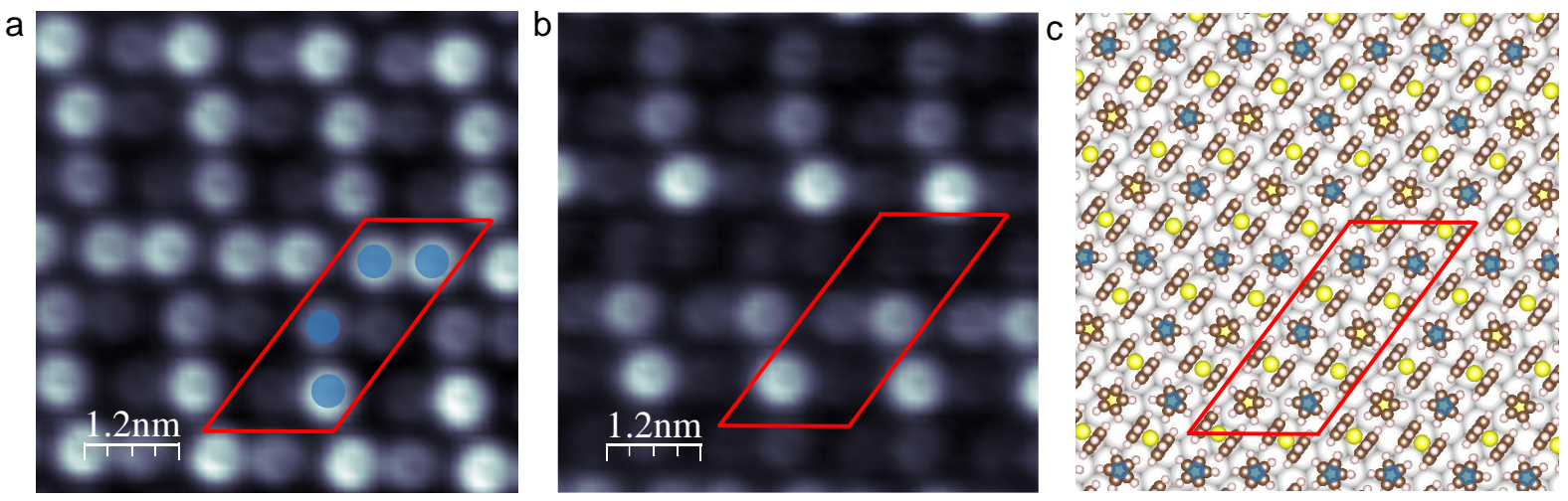

Figure 3: Molecular structure of the adsorbed islands with a clean superconducting tip. (a) Constant-height $d I / d V$ map taken at $6.6 \mathrm{mV}$, and (b) at $6.0 \mathrm{mV}$ over the paired phase, Fig. 2 (c). (c) Atomic structure of the unit cell for the aligned-dimer phase. ${ }^{46}$ The maps in (a) and (b) point at a unit cell formed by three vertical and three horizontal dimers aligned within an angle of $50^{\circ}$ with the [101] direction of the $\mathrm{Pb}(111)$ surface. The Nc molecules with the smallest computed MAE (HCP sites) are marked by a circle on the corresponding vertical molecule. The emerging pattern perfectly matches the $d I / d V$ map at $6.6 \mathrm{mV}$ in (a). The complementary pattern is also obtained and it is due to the molecules on the remaining top sites that present a lower MAE than on the HCP site.

The above results imply that the IETS of adsorbed molecules should depend on the adsorption site. In this way, an IETS study using a clean superconducting (absence of molecule) tip on molecular adlayers can actually yield important data for revealing the layer geometry. This is particularly pertinent for the case of weakly adsorbed molecules that are prone to incommensurate phases. ${ }^{15}$

We deposit $\mathrm{Nc}$ on $\mathrm{Pb}$ (111) to create ordered molecular islands (see Methods for details) and we find different adsorption phases. Figure 2 shows constant-current STM images of a representative set of molecular islands obtained on $\mathrm{Pb}(111)$. We find a linear arrangement of dimers, Fig. 2 (a) that largely coincides with the paired phase existing on $\mathrm{Cu}(100)$ and $\mathrm{Cu}$ (111). ${ }^{47}$ Another configuration presents a zig-zag pattern, Fig. 2 (b), where the Nc dimers 
alternate direction between rows. This can be clearly seen in the smaller-area images of Fig. 2 (c) and (d). Different combinations of the two arrangements can take place in the same island, either repeating one pattern or the other in different domains. We perform density functional theory (DFT) studies ${ }^{45}$ that show the two patterns share the same stabilization energy, compatible with the coexistence of both phases. The compact phase that appears on $\mathrm{Cu}(100)$ and $\mathrm{Cu}(111)^{47}$ are rare on $\mathrm{Pb}(111)$.

Similar to the IETS shifts for the molecular tip, the $d I / d V$ obtained with a clean superconducting tip on different sites of the island shows constant quasiparticle peaks, Fig. 2 (e) and (f), while the IETS peaks present a clear dependence on the measurement site of the molecular island, Fig. 2 (e) and (g). As for the molecular tip, we find three distinct peak energies that point at the molecule-substrate interaction as source of the dispersion of MAE values.

The split peaks display the same inter-peak distance as the peaks for different surface sites. These are 0.20 and $0.10 \mathrm{meV}$ for the adsorbed molecules, very similar to the 0.18 and $0.13 \mathrm{meV}$ shifts for the molecule on the tip. These data confirm our interpretation that for the molecular tip, the main change in MAE is due to the $\mathrm{Nc}$ adsorption on the tip. The interaction of the molecule with the surface is comparatively constant even as the molecule is pressed against the surface, as the lack of dependence on the current shows for large currents. We expect then that the molecular tip also gives information on the molecular adsorption on $\mathrm{Pb}(111)$.

The IETS spatial dependence is starker when mapping the molecular island at a bias matching one of the IETS peaks. Figure 3 shows two constant-height $d I / d V$ maps of Figure 2 (c). Panel 3 (a) presents the spatial distribution of the $d I / d V$ at $V=6.6$ and (b) at $V=6.0$ mV. Both maps, Fig. 3 (a) and (b), show a quasi-periodic pattern involving three dimers, instead of the single-dimer periodic structure of Fig. 2 (c).

In order to understand these spatial IETS peak patterns, Fig.3 (a) and (b), the location of the dimers on the substrate needs to be found. To do this, we perform STM images on 
the edge of the paired island to obtain the atomic resolution of the $\mathrm{Pb}$ (111) substrate. $^{45}$ From these data, the unit cell of Fig. 3 (c) emerges. As in previous studies, ${ }^{39,47,48}$ we find a combination of vertical (with the molecular axis perpendicular to the surface) and horizontal (parallel to the surface) Nc resulting from a T-shaped vdW interaction. ${ }^{45}$ The new pattern consists of two vertical molecules and two horizontal molecules. The experimental angle of the structure is $50^{\circ} \pm 4^{\circ}$, in good agreement with the angle of $53.5^{\circ}$ that optimizes a DFT structure based on 3 vertical and 3 horizontal dimers. ${ }^{45}$ This structure matches the experimental distances. A closer look shows that the theoretical structure does not contain top sites although in a strictly incomensurate lattice, rare events of one molecule falling on the top position is possible. The leading force behind the incommensurate structure is the difference between the van der Waals intermolecular distance and the lattice periodicity.

We further compute different molecular-layer energies by shifting the molecular structure over the surface. All the computed structures are within $20 \mathrm{meV}$ per molecule pointing out at the small molecule-surface interaction. As a consequence, the molecular structure presents some degree of incommensurability ${ }^{15}$ that translates in the quasi-periodicity of the IETS pattern Fig. 3 (a) and (b).

The two types of adsorbed molecules (vertical and horizontal) lead to different interactions with the substrate. The horizontal molecules are weakly interacting via vdW forces and their computed adsorption energy is $0.5 \mathrm{eV} /$ molecule. However, the vertical molecules do present hybridization between their frontier $\pi$ orbitals and the $s p$-electronic structure of the $\mathrm{Pb}(111)$ surface with $0.7 \mathrm{eV}$ of adsorption energy per molecule.

The $d I / d V$ patterns can be explained by realizing that there are no molecules adsorbing on the $\mathrm{Pb}$ top sites for the obtained structure. This reduces to basically two types of peaks, the shifts due to the FCC (same as bridge) and the shifts due to the HCP sites. The HCP sites are indicated with blue circles on Fig 3 (c). ${ }^{46}$ The pattern in Fig. 3 (b) takes place at the largest bias, that matches the HCP sites of our structure in good agreement with the larger shift found when the molecular tip probes HCP sites. 
The molecular adsorption pattern in the molecular islands is complex. The molecules sit on inequivalent sites on the surface, modulating their MAE, and they arrange in vertical and horizontal molecules. Their easy-axis anisotropy leads to a non-collinear arrangement of spins imposed by the molecular orientation. Our work shows that molecular adlayers of weakly bound molecules give a new strategy to create non-collinear spin textures using the local anisotropy of the molecules and their complex self-assembling properties. This study shows that depending on the adsorption site, different interactions will be at play changing the intrinsic MAE of the molecule. By doing a systematic study of substrates, it should be possible to extrapolate to a regime where the molecule-substrate interactions are negligible permitting us to infer the intrinsic molecular MAE.

In summary, we have used a Nc molecule on an STM tip to probe specific sites on a $\mathrm{Pb}$ (111) surface and hence measure the relative shift in MAE due to the molecule-surface interactions. With this knowledge, we have measured the MAE shifts for a molecular adlayer and revealed the adsorption conformation of a complex molecular structure formed by molecules in different orientations and positions. Given the strong MAE of these molecules, understanding the geometry permits us to predict a strong non-collinear spin arrangement on the surface. This is of interest for studying spin textures in low-dimensional systems as well as for inducing topological phases on superconducting surfaces.

\section{Acknowledgement}

Financial support from the Spanish MICINN (project RTI2018-097895-B-C44), the European H2020 FET open project MeMo (grant no. 766864) and the French Agence Nationale de la Recherche (grants no. ANR-13-BS10-0016, ANR-11-LABX-0058 NIE and ANR-10LABX-0026 CSC) are gratefully acknowledged. 


\section{Supporting Information Available}

Density functional calculation used to determine and characterize the molecular overlayer together with further experimental data.

\section{References}

(1) Mak, K. F.; Shan, J.; Ralph, D. C. Probing and controlling magnetic states in 2D layered magnetic materials. Nature Reviews Physics 2019, 1, 646-661, Number: 11 Publisher: Nature Publishing Group.

(2) Fert, A.; Cros, V.; Sampaio, J. Skyrmions on the track. Nature Nanotechnology 2013, 8, 152-156.

(3) Bergmann, K. v.; Kubetzka, A.; Pietzsch, O.; Wiesendanger, R. Interface-induced chiral domain walls, spin spirals and skyrmions revealed by spin-polarized scanning tunneling microscopy. Journal of Physics: Condensed Matter 2014, 26, 394002, Publisher: IOP Publishing.

(4) Udvardi, L.; Szunyogh, L.; Palotás, K.; Weinberger, P. First-principles relativistic study of spin waves in thin magnetic films. Phys. Rev. B 2003, 68, 104436.

(5) Bouaziz, J.; dos Santos Dias, M.; Ziane, A.; Benakki, M.; Blügel, S.; Lounis, S. Chiral magnetism of magnetic adatoms generated by Rashba electrons. New. J. Phys. 2017, 19,023010 .

(6) Hermenau, J.; Brinker, S.; Marciani, M.; Steinbrecher, M.; dos Santos Dias, M.; Wiesendanger, R.; Lounis, S.; Wiebe, J. Stabilizing spin systems via symmetrically tailored RKKY interactions. Nature Communications 2019, 10, 2565, Number: 1 Publisher: Nature Publishing Group. 
(7) Gambardella, P. et al. Supramolecular control of the magnetic anisotropy in twodimensional high-spin Fe arrays at a metal interface. Nature Materials 2009, 8, 189193, Number: 3 Publisher: Nature Publishing Group.

(8) Cuxart, M. G. et al. Molecular Approach for Engineering Interfacial Interactions in Magnetic/Topological Insulator Heterostructures. ACS Nano 2020, 14, 6285-6294, Publisher: American Chemical Society.

(9) Yosida, K. Theory of Magnetism; Springer Series in Solid-State Sciences; Springer Berlin Heidelberg, 2010.

(10) Sessoli, R.; Gatteschi, D.; Caneschi, A.; Novak, M. A. Magnetic bistability in a metalion cluster. Nature 1993, 365, 141-143, Number: 6442 Publisher: Nature Publishing Group.

(11) Amabilino, D. B. Supramolecular Chemistry at Surfaces; The Royal Society of Chemistry, 2016.

(12) Raval, R. Molecular assembly at surfaces: progress and challenges. Faraday Discussions 2017, 204, 9-33, Publisher: The Royal Society of Chemistry.

(13) Zobač, V.; Robles, R.; Lorente, N. Directionality in van der Waals Interactions: The Case of 4-Acetylbiphenyl Adsorbed on Au(111). The Journal of Physical Chemistry C 2020, 124, 4545-4551, Publisher: American Chemical Society.

(14) Franke, K. J.; Schulze, G.; Pascual, J. I. Competition of Superconducting Phenomena and Kondo Screening at the Nanoscale. Science 2011, 332, 940-944.

(15) Li, H. I.; Franke, K. J.; Pascual, J. I.; Bruch, L. W.; Diehl, R. D. Origin of Moiré structures in $C_{60}$ on $\mathrm{Pb}(111)$ and their effect on molecular energy levels. Phys. Rev. B 2009, 80, 085415 . 
(16) Malavolti, L.; Briganti, M.; Hänze, M.; Serrano, G.; Cimatti, I.; McMurtrie, G.; Otero, E.; Ohresser, P.; Totti, F.; Mannini, M.; Sessoli, R.; Loth, S. Tunable Spin-Superconductor Coupling of Spin 1/2 Vanadyl Phthalocyanine Molecules. Nano Letters 2018, 18, 7955-7961, PMID: 30452271.

(17) Farinacci, L.; Ahmadi, G.; Reecht, G.; Ruby, M.; Bogdanoff, N.; Peters, O.; Heinrich, B. W.; von Oppen, F.; Franke, K. J. Tuning the Coupling of an Individual Magnetic Impurity to a Superconductor: Quantum Phase Transition and Transport. Phys. Rev. Lett. 2018, 121, 196803.

(18) Barth, J. V.; Costantini, G.; Kern, K. Nanoscience and Technology; Co-Published with Macmillan Publishers Ltd, UK, 2009; pp 67-75.

(19) Clair, S.; de Oteyza, D. G. Controlling a Chemical Coupling Reaction on a Surface: Tools and Strategies for On-Surface Synthesis. Chemical Reviews 2019, 119, 4717-4776, Publisher: American Chemical Society.

(20) Guo, Y.; Nuermaimaiti, A.; Kjeldsen, N. D.; Gothelf, K. V.; Linderoth, T. R. TwoDimensional Coordination Networks from Cyclic Dipeptides. Journal of the American Chemical Society 2020, 142, 19814-19818, Publisher: American Chemical Society.

(21) Ho, W. Single-molecule chemistry. The Journal of Chemical Physics 2002, 117, 1103311061.

(22) Komeda, T. Chemical identification and manipulation of molecules by vibrational excitation via inelastic tunneling process with scanning tunneling microscopy. Progress in Surface Science 2005, 78, 41-85.

(23) Morgenstern, K.; Lorente, N.; Rieder, K.-H. Controlled manipulation of single atoms and small molecules using the scanning tunnelling microscope. physica status solidi (b) 2013, 250, 1671-1751. 
(24) Kim, Y.; Komeda, T.; Kawai, M. Single-Molecule Reaction and Characterization by Vibrational Excitation. Physical Review Letters 2002, 89, 126104, Publisher: American Physical Society.

(25) Bocquet, M.-L.; Lesnard, H.; Lorente, N. Inelastic Spectroscopy Identification of STMInduced Benzene Dehydrogenation. Physical Review Letters 2006, 96, 096101.

(26) Wegner, D.; Yamachika, R.; Zhang, X.; Wang, Y.; Crommie, M. F.; Lorente, N. Adsorption Site Determination of a Molecular Monolayer via Inelastic Tunneling. Nano Letters 2013, 13, 2346-2350.

(27) Chiang, C.-l.; Xu, C.; Han, Z.; Ho, W. Real-space imaging of molecular structure and chemical bonding by single-molecule inelastic tunneling probe. Science $\mathbf{2 0 1 4}, 344,885-$ 888, Publisher: American Association for the Advancement of Science Section: Report.

(28) Heinrich, A. J.; Gupta, J. A.; Lutz, C. P.; Eigler, D. M. Single-Atom Spin-Flip Spectroscopy. Science 2004, 306, 466-469.

(29) Gauyacq, J.-P.; Lorente, N.; Novaes, F. D. Excitation of local magnetic moments by tunneling electrons. Progress in Surface Science 2012, 87, 63-107.

(30) Ternes, M. Spin excitations and correlations in scanning tunneling spectroscopy. New J. Phys. 2015, 17, 063016.

(31) Czap, G.; Wagner, P. J.; Xue, F.; Gu, L.; Li, J.; Yao, J.; Wu, R.; Ho, W. Probing and imaging spin interactions with a magnetic single-molecule sensor. Science 2019, 364, $670-673$.

(32) Verlhac, B.; Bachellier, N.; Garnier, L.; Ormaza, M.; Abufager, P.; Robles, R.; Bocquet, M.-L.; Ternes, M.; Lorente, N.; Limot, L. Atomic-scale spin sensing with a single molecule at the apex of a scanning tunneling microscope. Science 2019, 366, 623-627. 
(33) Ji, S.-H.; Zhang, T.; Fu, Y.-S.; Chen, X.; Ma, X.-C.; Li, J.; Duan, W.-H.; Jia, J.-F.; Xue, Q.-K. High-Resolution Scanning Tunneling Spectroscopy of Magnetic Impurity Induced Bound States in the Superconducting Gap of Pb Thin Films. Phys. Rev. Lett. 2008, 100, 226801.

(34) Ruby, M.; Pientka, F.; Peng, Y.; von Oppen, F.; Heinrich, B. W.; Franke, K. J. Tunneling Processes into Localized Subgap States in Superconductors. Physical Review Letters 2015, 115, 087001.

(35) Choi, D.-J.; Rubio-Verdú, C.; de Bruijckere, J.; Ugeda, M. M.; Lorente, N.; Pascual, J. I. Mapping the orbital structure of impurity bound states in a superconductor. Nature Communications 2017, 8, 15175.

(36) Bastiaans, K. M.; Cho, D.; Chatzopoulos, D.; Leeuwenhoek, M.; Koks, C.; Allan, M. P. Imaging doubled shot noise in a Josephson scanning tunneling microscope. Physical Review B 2019, 100.

(37) Ormaza, M.; Bachellier, N.; Faraggi, M. N.; Verlhac, B.; Abufager, P.; Ohresser, P.; Joly, L.; Romeo, M.; Scheurer, F.; Bocquet, M.-L.; Lorente, N.; Limot, L. Efficient Spin-Flip Excitation of a Nickelocene Molecule. Nano Lett. 2017, 17, 1877-1882.

(38) Ormaza, M.; Abufager, P.; Verlhac, B.; Bachellier, N.; Bocquet, M. L.; Lorente, N.; Limot, L. Controlled spin switching in a metallocene molecular junction. Nat. Commun. 2017, 8, 1974.

(39) Bachellier, N.; Verlhac, B.; Garnier, L.; Zaldívar, J.; Rubio-Verdú, C.; Abufager, P.; Ormaza, M.; Choi, D.-J.; Bocquet, M.-L.; Pascual, J. I.; Lorente, N.; Limot, L. Vibronassisted spin excitation in a magnetically anisotropic molecule. Nature Communications 2020, 11, 1619 .

(40) Stroscio, J. A.; Celotta, R. J. Controlling the Dynamics of a Single Atom in Lateral Atom Manipulation. Science 2004, 306, 242-247. 
(41) Webb, G.; Marsiglio, F.; Hirsch, J. Superconductivity in the elements, alloys and simple compounds. Physica C: Superconductivity and its Applications 2015, 514, 17 - 27, Superconducting Materials: Conventional, Unconventional and Undetermined.

(42) Rolf-Pissarczyk, S.; Burgess, J. A. J.; Yan, S.; Loth, S. Closing the superconducting gap in small $\mathrm{Pb}$ nanoislands with high magnetic fields. Phys. Rev. B 2016, 94, 224504.

(43) Kezilebieke, S.; Zitko, R.; Dvorak, M.; Ojanen, T.; Liljeroth, P. Observation of Coexistence of Yu-Shiba-Rusinov States and Spin-Flip Excitations. Nano Letters 2019, 19, 4614-4619, PMID: 31251066.

(44) Mohr, M.; Gruber, M.; Weismann, A.; Jacob, D.; Abufager, P.; Lorente, N.; Berndt, R. Spin dependent transmission of nickelocene-Cu contacts probed with shot noise. Physical Review B 2020, 101, 075414.

(45) Please refer to the Supplemental Material.

(46) Momma, K.; Izumi, F. VESTA 3 for three-dimensional visualization of crystal, volumetric and morphology data. Journal of Applied Crystallography 2011, 44, 1272-1276, Number: 6 Publisher: International Union of Crystallography.

(47) Bachellier, N.; Ormaza, M.; Faraggi, M.; Verlhac, B.; Vérot, M.; Le Bahers, T.; Bocquet, M.-L.; Limot, L. Unveiling nickelocene bonding to a noble metal surface. Phys. Rev. B 2016, 93, 195403.

(48) Ormaza, M.; Abufager, P.; Bachellier, N.; Robles, R.; Verot, M.; Le Bahers, T.; Bocquet, M.-L.; Lorente, N.; Limot, L. Assembly of Ferrocene Molecules on Metal Surfaces Revisited. J. Phys. Chem. Lett. 2015, 6, 395-400. 